\title{
Analogies between Finite Difference and Finite Element Methods for Scalar and Vector Potential Formulations in Magnetic Field Calculations
}

\author{
Andrzej Demenko ${ }^{1}$ and Jan K. Sykulski² , Fellow, IEEE \\ ${ }^{1}$ Poznań University of Technology, Poznań, 60-965, Poland \\ ${ }^{2}$ Electronics and Computer Science, University of Southampton, Southampton, SO17 1BJ, United Kingdom
}

\begin{abstract}
Numerical 3D formulations using scalar $\Omega$ and vector $A$ potentials are examined for magnetic fields, with emphasis on the finite difference (FDM) and finite element (FEM) methods using nodal and facet elements. It is shown that for hexahedral elements the FDM equations may be presented in a form similar to the FEM equations; to accomplish this the coefficients defining volume integrals in FEM need to be expressed in an approximate manner, while the nodes in FDM require supplementary association with middle points of edges, facets and volumes. The analogy between a description of magnetic field sources arising from the classical $\mathrm{mm} f$ distribution approach and when expressed in terms of edge values of vector potential $T_{0}$ is emphasized. Comparisons are made between results obtained using FDM and FEM for both scalar and vector potential formulations. Forces in systems containing permanent magnets and torques in permanent magnet machines are calculated and compared using both approaches for scalar and vector formulations. A unified form of the stress tensor has been applied to FDM and FEM.
\end{abstract}

Index Terms - magnetic fields, finite difference methods, edge element method, finite element analysis.

\section{INTRODUCTION}

$\mathrm{T}$ HE OLDEST numerical routine for magnetic field modelling is the finite difference method (FDM); in its 2D guise it was likened to a classical finite element method (FEM) using nodal formulation [1]-[3] - there was some spirited debate at the time. The possibility to derive FDM from an energy functional similar to FEM was revealed in the discussion following [3]. In the classical FEM approach shape functions are employed; in the FDM the functional results from the definition of finite differences, where the average energy density in an element is a weighted average of values at nodes or at points between the nodes. Accordingly, the final equation differs from a typical FDM formulation only in the description of the average flux density. The aforementioned papers, however, considered only classical FEM approach and did not include the edge or facet formulations typical for 3D.

The authors of this paper have previously shown how suitable assumptions and approximations allowed the FEM equations to be identical to those obtained from the Finite Integration Technique (FIT) or equivalent reluctance networks [4], [5]. Analogies between edge formulations (EEM) in FEM and FDM were established when a magnetic vector potential $\boldsymbol{A}$ was employed [6]. The purpose of this paper is to extend the treatment to edge, facet and volume formulations of the FEM.

The ensuing discussion applies to formulations using the magnetic vector potential $\boldsymbol{A}$ for enforced current densities $\boldsymbol{J}$ or electric vector potential $\boldsymbol{T}$ for magnetization and conduction currents, but also magnetic scalar potential $\Omega$ under imposed

Manuscript received October 5, 2015; revised ??? ??, 2015 and ??? ??, 2015; accepted ??? ??, 2015. Date of publication ??? ??, 2015; date of current version ??? ??, 2015. Corresponding author: A. Demenko (e-mail: andrzej.demenko@put.poznan.pl).

Color versions of one or more of the figures in this paper are available online at http://ieeexplore.ieee.org.

Digital Object Identifier (inserted by IEEE). distribution of the vector potential $\boldsymbol{T}$. In the discussion about analogies it will be noted that in the classical FDM scheme field quantities are associated with nodes whereas in FEM may also be related to edges, facets or volumes.

The final section focuses on the generalized description appropriate for both FEM and FDM - of the stress tensor and associated computation of forces and torques. Results are compared between four methods: EEM and FDM using magnetic vector potential and FEM and FDM using scalar potentials. An example has been chosen for which an analytical solution exists for better assessment of accuracy.

\section{NODAL, EdGe, FACET AND VOLUME VALUES IN FDM}

Consider the 8-node region ( $P_{1}$ to $\left.P_{8}\right)$ depicted in Fig. 1 . In the 'standard' formulation of the FDM the field quantities are related to the nodes, whereas in FEM also to edges, facets and volumes. Therefore, when setting up equations for FDM equivalent to FEM, in addition to nodes $P_{i}$, ancillary points have been specified associated with the element's edges, facets and volume, positioned in the middle of the respective geometrical feature.

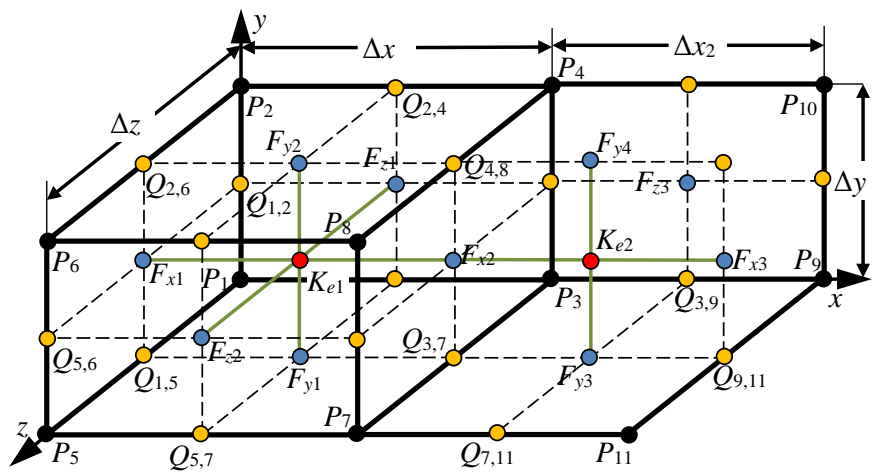

Fig. 1. Characteristic points for a hexahedral element.

At points in the middle of each edge the values of $\boldsymbol{A}, \boldsymbol{T}, \boldsymbol{H}$ 
and $\operatorname{grad} \Omega$ are defined, whereas in the middle of each facet also the values of $\boldsymbol{B}$ and $\boldsymbol{J}$. A simple relationship exists between the edge value $\varphi_{E i, j}$ of a vector $\boldsymbol{E}$ (where $\boldsymbol{E}=\boldsymbol{A}, \boldsymbol{T}, \boldsymbol{H}$ or $\operatorname{grad} \Omega$ ) for the edge $P_{i} P_{j}$ of the length $\Delta u$ (where $u=x, y$ or $z$ ) and the value $E_{u i, j}$ of the relevant $u$ component of $\boldsymbol{E}$ at the point $Q_{i, j}$, namely $\varphi_{E i, j}=\Delta u E_{u i, j}$. From this it is clear that the finite difference defined in FDM as $\left(\Omega_{j}-\Omega_{i}\right) \approx \Delta u \operatorname{grad} \Omega\left(Q_{i, j}\right)$ relates to the edge value of $\operatorname{grad} \Omega$ for the edge $P_{i} P_{j}$.

The facet value of a vector $\boldsymbol{S}(\boldsymbol{S}=\boldsymbol{J}, \boldsymbol{B}$ or $\boldsymbol{H})$ for a facet with the middle point $F_{u k}(u=x, y, z ; k=1,2, \ldots)$ may be expressed as a product of the area of that facet and the $u$ component of $\boldsymbol{S}$ at the point $F_{u k}$. For example, the relationship between the facet value $f_{y 1}$ of the vector $S$ and $S_{y 1}$, the value of a component of the vector $S_{y}$ at point $F_{y 1}$, may be written as $f_{y 1}=\Delta x \Delta z S_{y 1}$. In the dual finite element formulation a mesh has branches connecting the middle points $K_{e i}$ of adjacent volumes cutting a facet through its middle point $F_{u k}$ [7], [8]. Following [2], we will distinguish between the 'edge network' (EN) with nodes $P_{i}$ and the 'facet network' (FN) with nodes at $K_{e i}$. In the case of $\mathrm{FN}$, the volume value of potential $\Omega$ in an element is specified by the product of the potential $\Omega_{e i}$ in node $K_{e i}$ and the element volume. Moreover, the edge value of $\boldsymbol{S}$ for the edge $K_{e i} F_{u k}$ is given by the volume integral of the product of $\boldsymbol{S}$ and the interpolating function of the facet element for the facet with its middle point at $F_{u k}$. It can be shown that the edge value of $\operatorname{grad} \Omega$ is then equal to the difference between the average value $\Omega_{e i}$ of $\Omega$ in the volume and the average value $\Omega_{F u k}$ of $\Omega$ for the facet $F_{u k}$.

\section{NETWORK REPRESENTATIONS OF FEM AND FDM}

Analogies between FEM and equivalent magnetic networks are helpful. The FEM schemes relying on a scalar potential $\Omega$ and nodal elements are related to nodal equations of the permeance network, EN, with nodes $P_{i}$, whereas using a vector potential $\boldsymbol{A}$ and an edge formulation is equivalent to the loop equations of the reluctance network, FN, with nodes at $K_{e i}$ and loops around the edge $P_{i} P_{j}$ [4]. The loop fluxes $\varphi$ in FN represent the edge values of $\boldsymbol{A}$. However, the branch fluxes correspond to the facet values of flux density $\boldsymbol{B}$. The cycle (loop) matrix of the FN graph for paths around element edges represents the differential operator curl. The loop mmfs in FN are defined by facet values of the current density. The nodal values in the permeance EN represent the values of the potential $\Omega$ at nodes. The magnetic potential difference across a permeance of a branch assigned to an edge corresponds to the edge value of the magnetic field strength $\boldsymbol{H}$, while the branch $\mathrm{mmfs}$ relate to the edge values of the electric vector potential $\boldsymbol{T}$. The incidence matrix of the EN graph provides a network representation of the div operator.

In magnetic networks equivalent to FEM, couplings exist between branches of an element, i.e. mutual permeances $\Lambda_{i j, p q}$ between branches $P_{i} P_{j}$ and $P_{q} P_{p}$ in EN, or mutual reluctances $R_{\mu u k, r}$ between branches $K_{e i} S_{u k}$ and $K_{e i} S_{u r}$ in FN [4]. The relevant parameters may be calculated from

$$
\Lambda_{i j, p q}=\iiint_{V_{e}} \boldsymbol{w}_{e i j}^{T} \mu \boldsymbol{w}_{e p q} \mathrm{~d} \boldsymbol{v}, \quad R_{\mu u k, r}=\iiint_{V_{e}} \boldsymbol{w}_{f k}^{T} \mu^{1} \boldsymbol{w}_{f r} \mathrm{~d} \boldsymbol{v} \quad(1 \mathrm{a}, \mathrm{b})
$$

where $\boldsymbol{w}_{e i j}$ and $\boldsymbol{w}_{\text {epq }}$ are the interpolating functions of the edge element for the edge $P_{i} P_{j}, P_{p} P_{q}$, whereas $\boldsymbol{w}_{f k}$ and $\boldsymbol{w}_{f r}$ are the interpolating functions for the $k$ th and $r$ th facets of the facet element. For the parallelepiped element the functions take a relatively simple form. For example, if the edge $P_{p} P_{q}$ of an element with the center at $K_{e 1}$ is the edge $P_{1} P_{2}$, then

$$
\boldsymbol{w}_{\text {epq }}=\mathbf{1}_{y}(\Delta x-x)(\Delta z-z) / V_{e}
$$

For the $r$ th facet of the same element, positioned in the plane $x=0$ in Fig. 1, the interpolating function of the facet element is expressed by

$$
\boldsymbol{w}_{f r}=\mathbf{1}_{x}(\Delta x-x) / V_{e}
$$

As an illustration, the expressions for the self permeance $\Lambda_{15,15}$ in the branch coinciding with the edge $P_{1} P_{5}$ and mutual permeances $\Lambda_{15, p q}$ between the given branch and the branches associated with edges $P_{p} P_{q}$ are given by

$$
\Lambda_{15, p q}=0.25 v_{p} \mu \Delta z(\Delta x \Delta y)^{-1} \quad(p=1,2,3,4 ; q=p+4),
$$

where $v_{p}=4 / 9$ for the self permeance, i.e. for $p=1, q=5$, and $v_{p}=2 / 9$ for the mutual permeances between the branches $P_{1} P_{5}$ and $P_{2} P_{6}$, as well as between $P_{1} P_{5}$ and $P_{3} P_{7}$, that is for $p=2$, $q=6$ and $p=3, q=7$, or $v_{p}=1 / 9$ for the mutual permeances between the branches $P_{1} P_{5}$ and $P_{4} P_{8}$, i.e. $p=4, q=8$. The mutual permeances between the branch $P_{1} P_{5}$ and the remaining branches perpendicular to $P_{1} P_{5}$ are all equal to zero.

There are fewer mutual relationships in the reluctance facet model of an element. Applying (1b) to calculate the self and mutual reluctances for the branch $K_{e 1} F_{y 1}$ yields

$$
R_{\mu y 1, r}=v_{r} 0.5 \mu^{-1} \Delta y(\Delta z \Delta x)^{-1} \quad(r=1,2),
$$

where $v_{r}=2 / 9$ for the self reluctance, that is when $r=1$, while $v_{r}=-1 / 3$ in the case of the mutual reluctance between $K_{e 1} F_{y 1}$ and $K_{e 1} F_{y 2}$, i.e. $r=2$. It should be noted that the negative sign is a consequence of the assumed direction of the FN graph branches towards node $K_{e 1}$.

In FDM a grid is often used with nodes defined by the intersecting orthogonal lines in the cylindrical coordinates system. The elements of such a grid form ring sectors and are therefore curvilinear cuboids of orthogonal facets and edges. The parameters of such a model may also be derived from (1). However, due to the complexity of the interpolating functions when written in cylindrical coordinates it is usually helpful to introduce a local coordinate system and when deriving the integrals of (1) the Jacobian matrix is utilized. In such local coordinates $x^{\prime}, y^{\prime}$ and $z^{\prime}$ the relevant to (2) and (3) functions $\boldsymbol{w}_{i j}, \quad \boldsymbol{w}_{f r q}$ are then expressed as $\boldsymbol{w}_{e i j}=\mathbf{1}_{y}\left(1-x^{\prime}\right)\left(1-z^{\prime}\right)$; $\boldsymbol{w}_{f r}=\mathbf{1}_{x}\left(1-x^{\prime}\right)$, where the volume of the element is taken as unity.

The integrals (1) can be approximated as described in [5], i.e. using the following formula 


$$
\iiint_{V_{e}} f \mathrm{~d} v=V_{e} \frac{1}{N} \sum_{i=1}^{N} f\left(P_{i}\right)
$$

where $N$ is the number of element nodes and $f\left(P_{i}\right)$ is the value of the integrand $f$ at $P_{i}$. This results in the network equations for the hexahedral (cuboid) mesh being free of mutual terms, whereas self permeances or reluctances are described through simple relationships, e.g. for the branch $P_{1} P_{5}$ the permeance $\Lambda_{15,15}$ is

$$
\Lambda_{15,15}=0.25 \mu \Delta z(\Delta x \Delta y)^{-1},
$$

while for the branch $K_{e 1} F_{y 1}$ the reluctance $R_{\mu y 1,1}$ is defined as

$$
R_{\mu y 1,1}=0.5 \mu^{-1} \Delta y(\Delta z \Delta x)^{-1} .
$$

The absence of mutual terms makes the inversion of the branch parameters matrix of the equivalent meshes a much easier task. Thus the field distribution given by the edge values of $\boldsymbol{A}$ in FN may be found via a process of solving the equations for nodal potentials $\Omega_{e i}$. Similarly, the solution of equations describing the distribution of $\Omega$ at nodes $P_{i}$ of EN may be converted to a task of finding edge values of $\boldsymbol{A}$ for the edge $K_{e i} K_{e j}$. In this last transformation it is recognized that the edge value of $\boldsymbol{A}$ for $K_{e i} F_{u k}$ is related to the integral of the product $\boldsymbol{w}_{f k} \boldsymbol{A}$ and that $\boldsymbol{A}$ may be expressed in terms of the values for $P_{i} P_{j}$. In the language of circuit theory these transformations result in loop equations for $\mathrm{FN}$ being replaced by nodal equations and the nodal equations for EN by loop equations for loops assigned to element facets (see Fig. 2). (a)

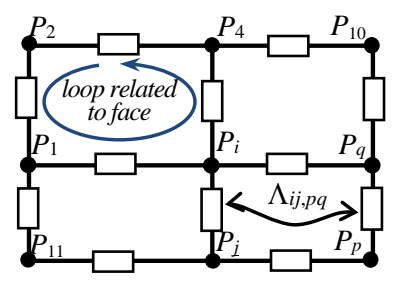

(b)

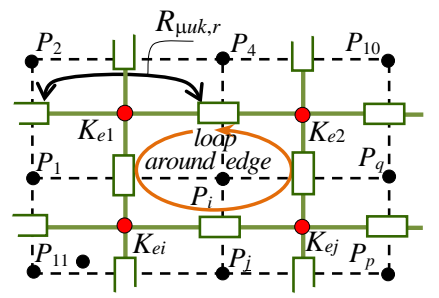

Fig. 2. Parts of equivalent magnetic networks: (a) edge EN, and (b) facet FN.

Similar models may be derived from FDM; if working with magnetic vector potential the procedures derived in [6] should be followed, thus assume the product of the $u$ component of $\boldsymbol{A}$ at point $Q_{i, j}$ and the edge length $\Delta u$ in the direction of $u$ to be the unknown, bearing in mind that the reluctivity at the center $F_{u i}$ of a facet is a weighted average of the volume values in elements attached to the facet. When using the scalar potential the permeability $\mu\left(Q_{i, j}\right)$ at the center of the edge $P_{i} P_{j}$ is taken as a weighted average of the four adjacent elements sharing the edge. When the energy functional is set up then inside an element $B^{2}=\Sigma_{u}\left(\Sigma_{i=1,2}\left(B_{u i}\right)^{2}\right) / 2, \quad H^{2}=\Sigma_{u}\left(\Sigma_{i, j}\left(H_{u i j}\right)^{2}\right) / 4$, where $B_{u i}=B_{u}\left(F_{u i}\right), H_{u i j}=H_{u}\left(Q_{i, j}\right), u=x, y, z$.

\section{REPRESENTATION OF SOURCES}

Sources may be described in two ways, either in terms of the imposed (prescribed) current density $\boldsymbol{J}$ using facet elements, or by working with edge elements and applying imposed (in the case of permanent magnets) or derived (e.g. from $\boldsymbol{J}=$ curl $\boldsymbol{T}$ ) distributions of electric vector potential $\boldsymbol{T}$ or $\boldsymbol{T}_{0}$. The former yields the loop $m m f s$, it is therefore only suitable for loop methods, e.g. using the magnetic vector potential $\boldsymbol{A}$, whereas the latter is more universal as from the edge values of $\boldsymbol{T}$ or $\boldsymbol{T}_{0}$ branch $\mathrm{mmfs}$ may be established thus making the description applicable to both nodal and loop methods, i.e. appropriate for derivations using either $\Omega$ or $\boldsymbol{A}$. Modern FEM formulations tend to use the latter description; it should be noted, however, that well before the advent of edge element formulations a version of this approach was already common in FDM, often referred to as the 'current linkage distributions' created by electrical machine windings.

The analogy between using the edge values of $\boldsymbol{T}_{0}$ and the concept of current linkage distributions may be explained with the aid of an example of a single turn in slots (Fig. 3). The turn has been replaced by five loops: $s_{1}$ and $s_{2}$ are associated with the $m m f$ exciting the slot leakage flux, $s_{3}$ and $s_{4}$ with the flux around the end connections, and $s_{5}$ with the air-gap main flux. A portion of the model for the $z=0$ plane is shown in Fig. 3b. In the classical FDM using scalar potential, where the sources are created on the basis of the current linkage distributions, the grid model uses the permeances with the branch $\mathrm{mmfs}$ equal to the current of the relevant turn. The FEM will yield an identical result providing (1a) is approximated using (6) and the sources are expressed in terms of the edge values $\boldsymbol{T}_{0}$ for the loop $s_{i}$. In the in-house software developed by the authors, used for analysis of the machine discussed in Section V, the edge values of $\boldsymbol{T}_{0}$ are established on the basis of the number of cuts of the element edges with the loops $s_{i}$. The purpose of this example was to show that the analogy between FDM and FEM extends to the description of field sources.

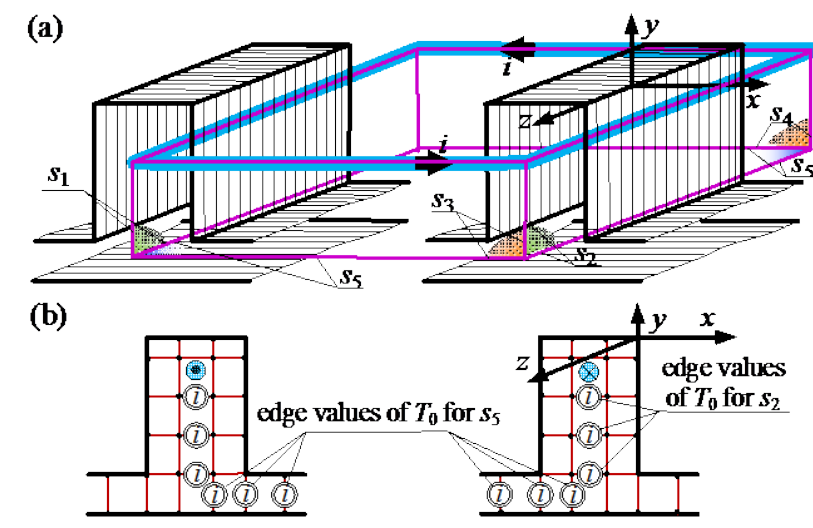

Fig. 3. A turn subdivided into loops (a), and branch $m m f \mathrm{~s}$ (b).

\section{COMPARISON OF FDM AND FEM - CASE STUdIES}

In design and engineering practice the magnetic field is usually needed to establish global parameters, such as losses, forces, stored energy, etc. Let us consider the calculation of attractive and repulsive forces in a 'test' system containing permanent magnets and torque in a permanent magnet machine. The first case concerns three magnets 'suspended' in the air as shown in Fig. 4 (a similar system, but of different dimensions, was analyzed in [9]). The permeability of the 
magnets was assumed to be $\mu_{0}$, while the magnetization perpendicular to their 'upper' surface. PM II and PM III have the same direction of magnetization which is either the same or opposite to PM I. Under the above assumptions the forces may be found analytically [10] so that the accuracy of numerical calculations can be assessed.

Figure 4 shows the force $F_{x}$ acting on PM I as a function of the distance $\Delta w$ between the magnets PM II and PM III, while other dimensions were fixed as $l=w=\Delta w+2 w_{c}, \delta=h=w / 3$. The force itself is taken as a relative value $F_{r}=F_{\chi} /\left(w l \mu_{0}\left(H_{c}\right)^{2}\right)$. Five different algorithms have been tried to compute the field distribution; two used the edge values of the vector potential $\boldsymbol{A}$, whereas the other three relied on the nodal values of the scalar potential $\Omega$. Space has been meshed using hexahedral elements and homogenous elements have been applied to the magnets with $\Delta y=\Delta z=\Delta x=w / 24$. Overall, there are just over $5.5 \times 10^{5}$ hexahedral elements and the resulting mesh is referred to as HeN. In an alternative formulation each hexahedron is divided into five tetrahedrons; the mesh is then described as TeN and contains over $2.7 \times 10^{6}$ elements. As in TeN the number of edges is more than double that in $\mathrm{HeN}$, it was decided not to compare the effectiveness of EEM for both types of meshes (due to anticipated long computing times); calculations for TeN were done only for the nodal formulation as computing times for TeN and HeN are comparable.

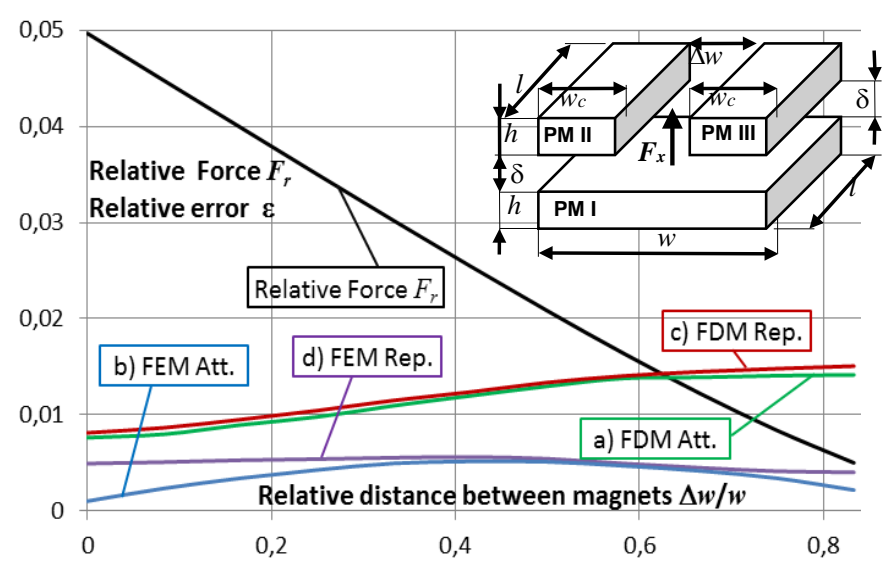

Fig. 4. Relative force and relative errors in force calculations using the edge value of $\boldsymbol{A}$ and $\mathrm{HeN}$ (hexahedral elements), $\varepsilon=\left(F_{\text {rexact }}-F_{r}\right) / F_{\text {rexact }}$.

In view of the particular algorithm used for the computation of forces, the EEM equation for the ith edge of the mesh has been written as

$$
\sum_{k} \sum_{j} \Theta_{i, j}^{(k)}=\theta_{i}
$$

where $\theta_{i}$ is the RHS of the edge element equation, and $\Theta_{i, j}^{(k)}$ is the $m m f$ ('magnetic voltage') in the jth branch of the loop around the ith edge, i.e. in the branch connecting the center of the $j$ th facet with the center $K_{e k}$ of the $k$ th element. As an example, for the edge $P_{1} P_{3}$ and the facet with the center at $F_{y 1}$ of the element of Fig. 1, the relevant $m m f$ reads

$$
\Theta_{i, j}=\Theta\left(K_{e 1}, F_{y 1}\right)=0.5 \mu^{-1} \Delta y(\Delta z \Delta x)^{-1}\left[v_{1} \phi\left(F_{y 1}\right)+v_{2} \phi\left(F_{y 2}\right)\right],
$$

where $\phi\left(F_{y i}\right)(i=1,2)$ is the facet value of the flux density for the facet with a center at $F_{y i}$, while $v_{i}$ denotes a weight, with $v_{1}=1$, $v_{2}=0$ for FDM and $v_{1}=2 / 3, v_{2}=-1 / 3$ for FEM, i.e. for a model with mutual reluctances. In EEM formulation, $\phi\left(F_{y i}\right)$ is expressed by edge values of $\boldsymbol{A}$, i.e. by loop fluxes $\varphi_{p}$ around edges, $\phi\left(F_{y i}\right)=\Sigma_{p} \varphi_{p}$.

Applying the virtual work principle to this formulation of EEM, as explained in [9], results in particularly suitable formulae for average values of the stress tensor for elements. As an example, for an element with the center at $K_{e 1}$, the mean value of the $T_{z z}$ component of the tensor may be expressed by

$$
T_{z z}=\frac{0.25}{\Delta z \Delta x \Delta z}\left(\Theta_{z 2} \phi_{z 1}+\Theta_{z 1} \phi_{z 2}-\Theta_{x 1} \phi_{x 1}-\Theta_{x 2} \phi_{x 2}-\Theta_{y 1} \phi_{y 1}-\Theta_{y 2} \phi_{y 2}\right),
$$

where $\Theta_{u i}=\Theta\left(K_{e 1}, F_{u i}\right), \phi_{u i}=\phi\left(F_{y i}\right),(u=x, y, z, i=1,2)$.

In the scalar potential approach for FDM and FEM, the equation for the node $P_{i}$ have been expressed as follows

$$
\sum_{k} \sum_{j} \phi_{i, j}^{(k)}=0
$$

where $\phi_{i, j}^{(k)}$ is the magnetic flux in the branch $P_{i} P_{j}$ for the $k$ th element. As an example, for the branch associated with $P_{1} P_{5}$ in HeN (see Fig. 1)

$$
\phi_{1,5}=0.25 \mu \Delta z(\Delta x \Delta y)^{-1} \sum_{i=1}^{4} v_{i}\left(\Omega_{i+4}-\Omega_{i}\right),
$$

where $\Omega_{i}$ and $\Omega_{i+4}$ are the nodal values of $\Omega$ in $P_{i}$ and $P_{i+4}$, respectively, and $v_{i}$ is the weight, with $v_{1}=1, v_{2}=v_{3}=v_{4}=0$ for FDM and $v_{1}=4 / 9, v_{2}=v_{3}=2 / 9, v_{4}=1 / 9$ for FEM, i.e. for the model with mutual permeances. Similar expressions were used in TeN, although more branches were associated with each node and different weights $v_{i}$ were prescribed.

The adopted description of terms in (14) has been applied to force calculations. In a similar way as in the case of EEM, the principles established in [9] have been followed making the algorithms for force and torque estimation consistent with the peculiarities of FEM and FDM formulations. In the resultant equations the mean value of $T_{z z}$ for the element with a center $K_{e 1}$ is given by

$$
T_{z z}=0.5(\Delta z \Delta x \Delta y)^{-1} \sum_{i=1}^{4}\left(\phi_{i} \Omega_{i+4}+\phi_{i+4} \Omega_{i}\right),
$$

where $\phi_{i}$ and $\phi_{i+4}$ describe the resultant flux through element branches towards nodes $P_{i}$ and $P_{i+4}$, respectively. The other components of the stress tensor are derived in a similar way.

Expressions (9) and (14) may be considered as generalized formulae originally suggested in [9] and for a mesh of homogeneous parallelepiped elements yield results equivalent to the application of the Lorentz method for systems with magnetizing currents, for both FDM and FEM. It should be noted, however, that although (9) and (14) look identical for FDM and FEM, expressions for $\Theta_{u i}$ and $\phi_{i, j}^{(k)}$ are not the same due to different weights applied to the formulations.

For the HeN approach, the relative errors in the attractive (Att) and repulsive (Rep) force calculations are shown in Figs. 4 and 5, with curves (a) and (c) referring to FEM, while (b) and (d) to FDM.

A detailed analysis of the TeN results revealed that expression (14) is also suitable for meshes with tetrahedral 
elements. However, the tensor method based on (14) and the Lorentz approach give appreciably different results. Therefore in Fig. 6 the errors in the Lorentz method (marked 'Lor') are also shown.

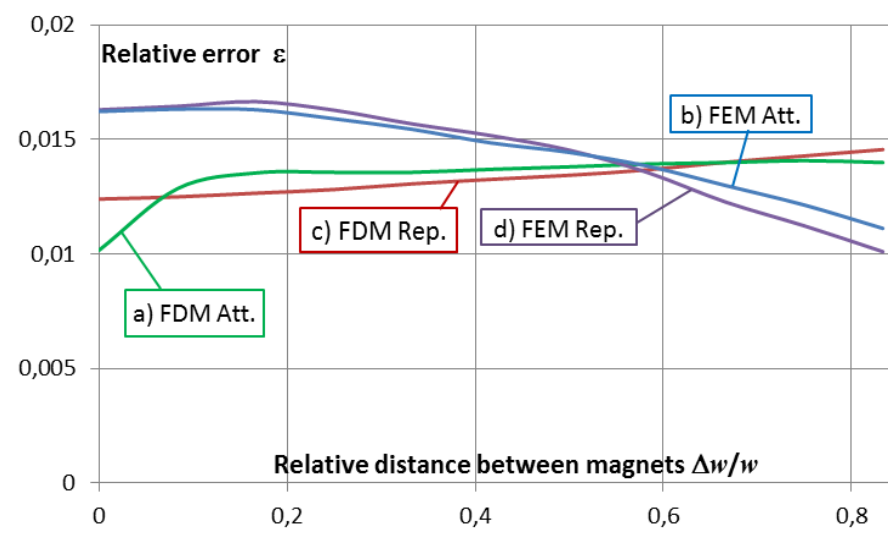

Fig. 5. Relative errors in force calculations for HeN using scalar potential.

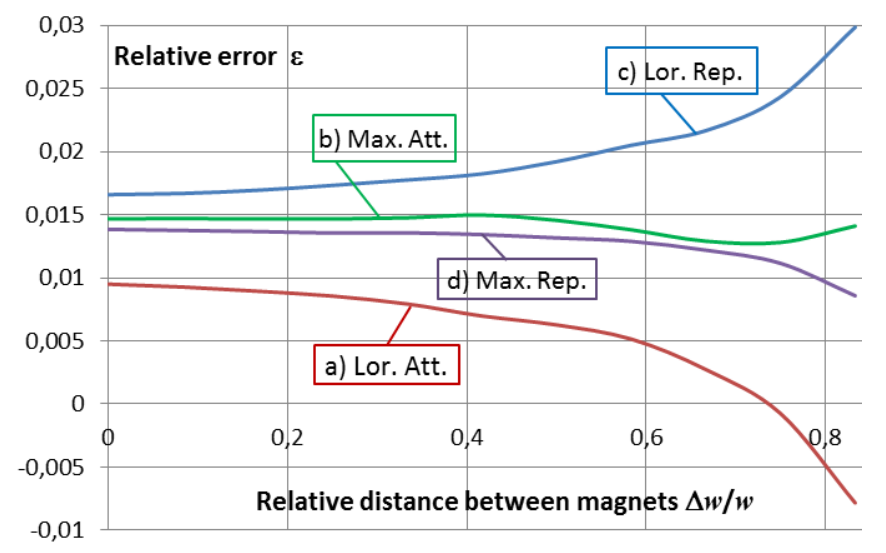

Fig. 6. Relative errors in force calculations for TeN using scalar potential.

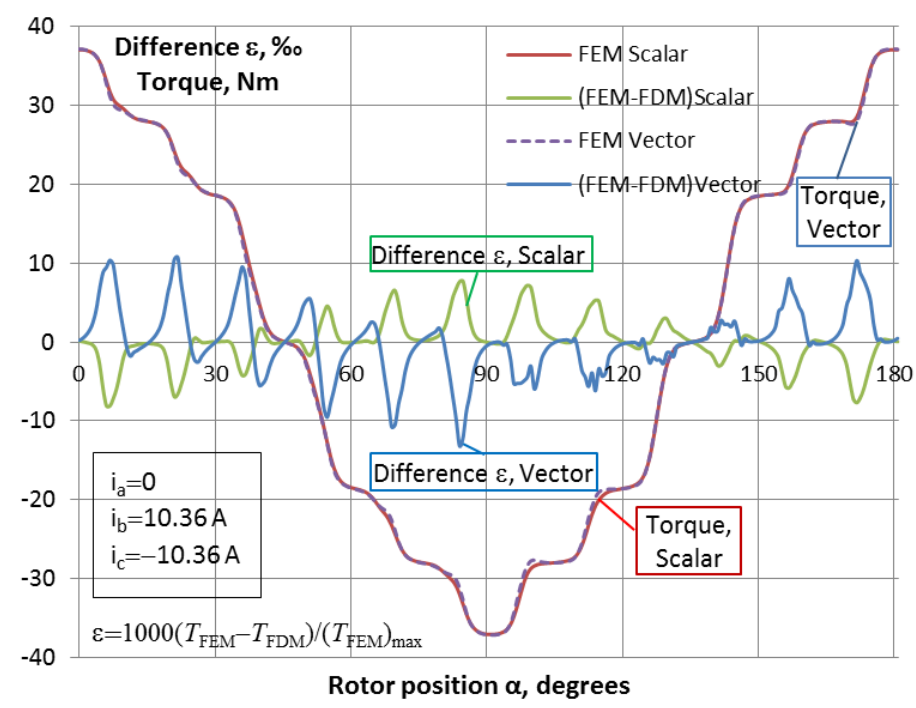

Fig. 7. Torque calculated using FEM for scalar and vector potential methods and relative difference $\varepsilon$ (in permillage \%o, that is per thousand) between results from FEM and FDM.

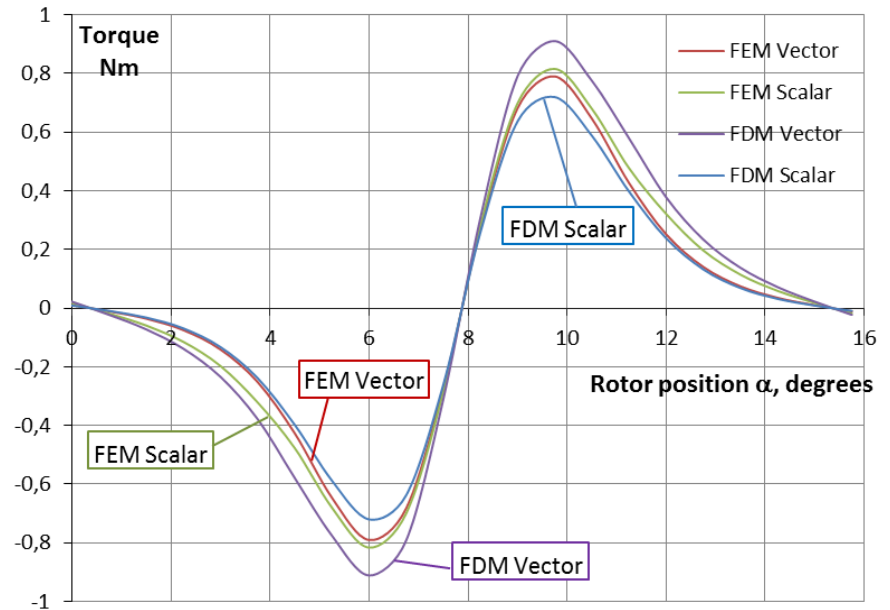

Fig. 8. Calculations of the cogging torque for a specific motor [11].

The comparisons of torque calculations are illustrated using a permanent magnet motor described in [11]. Curvilinear cuboidal elements were used in polar coordinates, which in the slot region were supplemented by triangular curvilinear prisms of 3 edges parallel to the machine shaft axis (for better representation of the slot shape), thus the types of elements unusual for the FDM. The model for such a type of prisms was introduced in [5]. The FEM parameters were then established directly using accurate expressions (1), whereas in the case of FDM formula (6) was used.

In torque calculations expressions similar to (9) and (14) were used. Figure 7 shows calculated torque at different rotor positions under imposed winding currents. As emphasized by Fig. 8, the differences between FDM and FEM are mainly in the calculated cogging torques. The superiority of FEM should be noted as the results for both potentials are similar, unlike in FDM. For the case with permanent magnets a better accuracy of FEM is exhibited only for a larger separation $\Delta w$ between the magnets (Fig. 5). The results endorse the need to reconcile the methodologies for force and torque calculation with the filed solution itself.

\section{CONCLUSION}

The equivalence between finite difference (FDM) and finite element (FEM) formulations, under certain assumptions, has been demonstrated. For rectangular parallelepiped, when appropriate approximations are applied to integrals arising from the FEM formulation, equations suitable to FDM emerge for points associated not only with element nodes but also with edges and facets. The analogy also embraces the representation of field sources defined by the edge values of $\boldsymbol{T}_{0}$. For both methods a similar form of the stress tensor may be used, as explained by relevant equations ((5) and (8)). Extensive numerical experiments have not demonstrated any particular advantage of FEM over FDM. Potential benefits will occur, however, for complicated material boundaries, difficult to represent in classical FDM where grid refinement would normally be necessitated. The use of inhomogeneous grid in FEM, on the other hand, often results in poorer accuracy of force and torque calculations, as exemplified by 
the cases studied - see results of force calculation for mesh of tetrahedral elements. A significant observation is that by using appropriate formulae almost identical results may be expected in force and torque calculations for scalar and vector formulations considered in this article. Even though these days the FDM is less popular (except in high frequency modelling) it is to be expected that as computational power increases a fine finite difference grid will be able to represent even complicated shapes thus the interest in the method may be on the rise.

\section{REFERENCES}

[1] N. A. Demerdash and T. W. Nehl, "An evaluation of the methods of finite elements and finite differences in the solution of nonlinear electromagnetic fields in electrical machines," Trans. Power App. Syst., vol. 98, no. 1, pp. 74-87, 1979.

[2] E. F. Fuchs and G. A. McNaughton, "Comparison of Fist-Order Finite Difference and Finite Element Algorithms for the Analysis of Magnetic Fields. Part I: Theoretical Analysis,” Trans. Power App. Syst., vol. 101, no. 5, pp. 1170-80, 1982.

[3] G. A. McNaughton and E. F. Fuchs, "Comparison of Fist-Order Finite Difference and Finite Element Alogorithms for the Analysis of Magnetic Fields. Part II: Numerical Examples,” Trans. Power App. Syst., vol. 101, no. 5, pp. 1181-1201, 1982.

[4] A. Demenko and J. K. Sykulski, "Network Equivalents of Nodal and Edge Elements in Electromagnetics," IEEE Trans. Magn., vol. 38 no. 2, pp. 1305-08, 2002.

[5] A. Demenko, J. K. Sykulski, and R. Wojciechowski, "On the Equivalence of Finite Element and Finite Integration Formulations,” IEEE Trans. Magn., vol. 46, no. 8, pp. 3169-72, 2010.

[6] A. Demenko and J. K. Sykulski, "On the equivalence of finite difference and edge element formulations in magnetic field analysis using vector potential,” COMPEL, vol. 33, No. 1/2, pp. 47-55, 2014.

[7] Z. Ren and Qu Hui, "Investigation of the Complementarity of Dual Eddy Current Formulations on Dual Meshes," IEEE Trans. Magn., vol. 46 no. 8, pp. 3161-64, 2010.

[8] Z. Ren, "On the complementarity of dual formulation on dual meshes," IEEE Trans. Magn., vol. 45, no. 3, pp. 1284-1287, Mar. 2009.

[9] A. Demenko, W. Lyskawinski, and R. M. Wojciechowski, "Equivalent Formulas for Global Magnetic Force Calculation from Finite Element Solution,” IEEE Trans. Magn., vol. 48, no. 2, pp. 195-198, 2012.

[10] H. Allag and J. P. Yonnet, "3-D analytical calculation of the torque and force exerted between two cuboidal magnets," IEEE Trans. Magn., vol. 45, no. 10, pp. 3969-3972, Oct. 2009.

[11] A. Demenko, R. M Wojciechowski, and J. K. Sykulski, "2D Versus 3D Electromagnetic Field Modeling in Electromechanical Energy Converters”, IEEE Trans. Magn., vol. 50, no. 2, art 7022204, 2014. 